\title{
Ketamine for the Treatment of Chronic Pain: A Comprehensive
}

\section{Review}

Jacob E. Israel, MD1, Samantha St Pierre, BS², Emily Ellis, BS², Josephine S. Hanukaai, BS'1, Nazir Noor, MD³, Giustino Varrassi, MD, PhD, FIPP ${ }^{4}$, Markus Wells, MD ${ }^{1}$, Alan D. Kaye, MD, Ph.D. ${ }^{5}$

1 School of Medicine, Georgetown University, ${ }^{2}$ School of Medicine, Louisiana State University Health Shreveport, ${ }^{3}$ Mount Sinai Medical Center, 4 Paolo Procacci Foundation, 5 Department of Anesthesiology, Louisiana State University Health Shreveport

Keywords: depression, post-thoracotomy pain syndrome, cancer pain, fibromyalgia, phantom limb syndrome, complex regional pain syndrome, neuropathic pain, ketamine, chronic pain

https://doi.org/10.52965/001c.25535

\section{Health Psychology Research}

Vol. 9, Issue 1, 2021

\section{Background}

Chronic pain significantly worsens the quality of life. Unlike neuropathic, musculoskeletal, postoperative pain, and cancer pain, chronic primary pain cannot be explained by an underlying disease or condition, making its treatment arduous.

\section{Objectives}

This manuscript intends to provide a comprehensive review of the use of ketamine as a treatment option for specific chronic pain conditions.

\section{Study Design}

A review article.

\section{Setting}

A review of the literature.

\section{Methods}

A search was done on PubMed for relevant articles.

\section{Results}

A comprehensive review of the current understanding of chronic pain and the treatment of specific chronic pain conditions with ketamine.

\section{Limitations}

Literature is scarce regarding the use of ketamine for the treatment of chronic pain.

\section{Conclusion}

First-line treatment for many chronic pain conditions includes NSAIDs, antidepressants, anticonvulsants, and opioids. However, these treatment methods are unsuccessful in a subset of patients. Ketamine has been explored in randomized controlled trials (RCTs) as an alternative treatment option, and it has been demonstrated to improve pain symptoms, patient satisfaction, and quality of life. Conditions highlighted in this review include neuropathic pain, fibromyalgia, complex regional pain syndrome (CRPS), phantom limb pain (PLP), cancer pain, and post-thoracotomy pain syndrome. This review will discuss conditions, such as neuropathic pain, fibromyalgia, complex regional pain syndrome, and more and ketamine's efficacy and its supplementary benefits in the chronic pain patient population. As the opioid crisis in the United States continues to persist, this review aims to understand better multimodal analgesia, which can improve how chronic pain is managed.

\section{INTRODUCTION}

Chronic pain is discomfort that continues beyond the usual timeframe for healing, becoming classified as chronic when it persists for longer than three months. It is a multifactorial issue, affecting roughly $20 \%$ of the world's population. Chronic pain is responsible for over $15 \%$ of patient visits and is associated with significant disruptions in the 
quality of life and emotional well-being. ${ }^{1}$ Chronic pain patients with resulting limited activity are also more likely to have comorbid conditions such as depression, COPD, and diabetes. ${ }^{2}$ There are many types of chronic pain, generally categorized between primary and secondary pain. Chronic primary pain cannot be explained by another condition or disease process. In contrast, other forms of chronic pain include neuropathic, musculoskeletal, cancer, postsurgical or posttraumatic, and visceral pain. ${ }^{3}$ Despite being a common condition, treatment for chronic pain has been challenging. Pharmacological, procedural, and behavioral interventions vary depending on chronic pain type, but many patients fail to experience a significant pain reduction. For example, randomized clinical trials (RCTs) studying pharmacotherapy in chronic neuropathic pain found that less than half of patients experience clinically significant pain relief. ${ }^{4}$ Furthermore, many chronic pain treatments have concerning adverse effects, particularly opioids. ${ }^{5}$

\section{EPIDEMIOLOGY}

In the United States alone, as many as $34.7 \%$ of females and $26.7 \%$ of males experience chronic pain. ${ }^{2}$ A survey in India also found that $63 \%$ of respondents reported their pain as severe rather than mild or moderate amongst patients with chronic pain. ${ }^{6}$ The socio-demographic, psychological, and genetic risk factors can all play a role in determining chronic pain outcomes for patients worldwide. ${ }^{7}$ In a 2016 survey conducted by the National Center for Health Statistics, high-impact chronic pain was associated with increasing aging. When corrected for age, the highest prevalence of chronic pain was found in previously employed women, those living in or near poverty, and those living in rural areas.

In contrast, the prevalence was lower among adults with at least a bachelor's degree. ${ }^{8}$ While the exact mechanism of chronic pain has not yet been elucidated, studies have demonstrated a genetic link to certain chronic pain states. ${ }^{7,9}$ Chronic pain can ultimately affect many aspects of an individual's mental, emotional, and physical well-being. Chronic pain patients report detrimental changes to their quality of life, such as the inability to exercise, poor sleep, and more difficulty maintaining relationships with others. ${ }^{6}$ As a result, it has been found that $20-50 \%$ of chronic pain patients have comorbid depression. ${ }^{10}$ Chronic pain also has a marked financial impact on society, costing $€ 200$ billion annually in Europe and $\$ 150$ billion annually in the United States. ${ }^{7}$

\section{RISK FACTORS}

As a condition with many etiologies, there are many risk factors associated with chronic pain. A well-established factor is increased age. With aging, individuals are more likely to acquire other comorbidities that can lead to or exacerbate chronic pain. ${ }^{10}$ Studies have found that being female is another risk factor. Females reportedly have different analgesic sensitivity compared to men. This includes lower pain tolerance, lower pain thresholds, and higher intensity sensation to noxious stimuli. ${ }^{7,11}$ In addition, environmental and behavioral risk factors may play a role in chronic pain states. Inadequate vitamin-D levels pose a risk factor for chronic pain as low levels are suggested to cause endocrine, neurological, and immunological changes that can affect chronic pain. ${ }^{10}$ The National Comorbidity survey reported that children who have been victims of abuse are also more likely to develop chronic pain, as both abuse and pain are linked to depression. ${ }^{12}$ Additionally, the presence of other morbidities increases the risk of experiencing chronic pain. After adjusting for socioeconomic factors, studies have shown an increased co-occurrence of chronic pain with cardiovascular disease, depression, cancer, neurological conditions, and chronic pulmonary disease. ${ }^{10}$ Chronic pain can also affect these comorbidities, as patients who report severe chronic pain are twice as likely to die from ischemic heart disease and COPD than those without chronic pain. ${ }^{7}$ Finally, sleep disorders have been shown to have a bidirectional effect on chronic pain, in that chronic pain causes poor sleep, and poor sleep increases the level and duration of pain. ${ }^{10,13}$

\section{TYPES OF CHRONIC PAIN}

The International Association for the Study of Pain (IASP) contacted the World Health Organization (WHO) and established a Task Force for the Classification of Chronic Pain. Temporally, they define chronic pain as pain that lasts or recurs for a period longer than three months. Chronic primary pain describes pain in one or more anatomic regions that another condition cannot explain, meant to better classify a large subset of chronic pain with an unknown etiology. Other subsets of chronic pain pertain to another primary condition or known insult, of which pain is a symptom. This includes chronic cancer-related pain, chronic postsurgical or posttraumatic pain, chronic neuropathic pain, chronic headache or orofacial pain, chronic visceral pain, and chronic musculoskeletal pain. ${ }^{3}$ Determining the correct type of chronic pain in a patient is essential. Different etiologies and resultant diagnoses correspond to different treatment practices, and individual circumstances may further guide the proper course of care.

\section{PATHOPHYSIOLOGY OF CHRONIC PAIN}

The nociceptive system serves a vital protective function for the human body. It is responsible for sensing and processing noxious stimuli and ultimately potentiating a response to possible physiological threats. Noxious mechanical and thermal stimuli and endogenous and exogenous chemical mediators activate nociceptors, which in turn trigger pathways that produce the subjective experience of pain. ${ }^{14}$ The nociceptive system is dynamic and has peripheral and central mechanisms to modulate sensitization to stimuli. ${ }^{15} \mathrm{Ex}-$ amples of this adaptability include peripheral and central sensitization and central modulation. Chronic pain is the dysfunction of this nociceptive system and negates the purpose of pain as a marker of potential immediate physiologic injury. ${ }^{16}$

Central modulation of pain is a descending network of endogenous mechanisms within the central nervous system 
(CNS) that diminish pain through net inhibition. ${ }^{17}$ Studies have demonstrated that neurons within the rostral ventromedial medulla (RVM) can change the experience of pain by modulating nociceptive traffic through their projections to the medullary or spinal dorsal horns. ${ }^{18}$ Although the exact mechanism is unknown. The descending pathway is said to be opiate sensitive given that pain-relieving drugs, such as opiates and NSAIDs, have been shown to mimic or enhance the modulatory circuit's effects at the spinal cord level. ${ }^{18}$

Sensitization is the prolonged but reversible increase in excitability and synaptic efficacy of neurons in nociceptive pathways triggered by nociceptor stimulation. ${ }^{15}$ Examples of this are allodynia, pain in the presence of a stimulus that usually does not trigger pain, and hyperalgesia increased pain due to a stimulus that already provokes pain. ${ }^{15}$ At the molecular level, these changes are seen both centrally and peripherally. Peripheral sensitization occurs at the level of the nociceptor and is marked by an increase in transcription, translation, and insertion of transducer channels and voltage-gated ion channels. ${ }^{14}$ Central sensitization refers to synaptic plasticity were alterations in ion channel kinetics and properties, increased density of ionotropic receptors, and activation of kinases both pre-and post-synaptically allow for amplification of nociceptive signaling. ${ }^{14}$

N-methyl-D-aspartate (NMDA) receptors, particularly those with the NR2B subunit, are believed to be a central component of this increased excitability. ${ }^{19}$ Studies have demonstrated increased peripheral expression of NMDA receptors as a consequence of tissue and nerve injury. ${ }^{20} \mathrm{Cen}$ trally, the composition of NMDA subunits within the spinal cord has been found to change due to increased nociceptive signaling - with the expression of NR2B subunit preferentially increased. ${ }^{21}$ The pathway for increased expression and composition of NMDA receptors is not entirely understood but is believed to involve possible transcriptional, translational, and posttranslational modulation secondary to nociceptor stimulation. ${ }^{19}$

The ICD-11 organizes chronic pain into seven clinical subgroups based on etiology; however, the pathophysiology of chronic pain shares the common theme of increased sensitivity irrespective of the initial physiologic assault. ${ }^{1,14}$ While acute pain is the result of nociceptor signaling in the presence of noxious stimuli. Chronic pain is believed to result from continued signaling, even in the absence of a stimulus, due to increased sensitivity. ${ }^{14}$ Peripheral and central sensitization of the nociceptive system is thereby believed to be crucial to the pathophysiology of chronic pain since these networks amplify clinical pain symptoms even in the reduced presence or absence of nociceptor stimulation. ${ }^{19}$ Pharmacological therapies that can target these sensitization pathways, such as blocking NMDA receptors, may be influential in tackling the treatment of chronic pain.

\section{HISTORY AND GENERAL CLINICAL APPLICABILITY OF KETAMINE}

Ketamine was synthetically developed in 1962 and was first tested clinically as a fast-acting general anesthetic in prisoners two years later. ${ }^{22}$ At the time, it was hoped that ketamine's short half-life and relatively minor behavioral tox- icity would allow it to become a replacement for phencyclidine (PCP), which was removed from the US market in $1965 .{ }^{23}$ Ketamine was approved by the FDA in 1972 and quickly became popular as a battlefield anesthetic in the Vietnam War.

In the decades that followed, the drug waned in usage as an anesthetic due to its adverse psychomotor effects. ${ }^{22}$ These side effects, such as delirium, dysphoria, and hallucinations, along with the drug's emergence as a popular recreational drug, lead the DEA to classify ketamine as a Schedule III Controlled Substance in $1999 .{ }^{24}$ The drug instead became a popular veterinary anesthetic, and in humans became more often used as an anesthetic for pediatric patients than adults. ${ }^{25}$

In recent years, interest in the therapeutic uses of ketamine has broadened to include possible analgesic and psychiatric indications. ${ }^{25}$ In emergency rooms across the country, ketamine has become a drug of choice to treat acute pain refractory to standard analgesic regimens or in patients with opioid dependence. ${ }^{26}$ In chronic pain conditions, such as mixed neuropathic pain, complex regional pain syndrome (CRPS), and phantom limb pain, ketamine is an effective long-term treatment option. ${ }^{27}$ Compelling evidence has also been found in seven placebo-controlled, double-blind, randomized clinical studies of possible antidepressant effects of ketamine. ${ }^{28,29}$

Despite recent discoveries, ketamine remains only approved by the FDA as an anesthetic agent for surgical and diagnostic procedures. ${ }^{30}$ Since ketamine is widely available, inexpensive, and generic - it is unlikely and cost-prohibitive that any new indication will be approved in the years ahead. $^{25}$ Nevertheless, off-label usage and research will likely continue to grow as ketamine becomes a more widely sought-after therapeutic agent for chronic pain, mood disorders, and refractory pain.

\section{MECHANISM OF ACTION}

Ketamine is a chiral arylcyclohexylamine derivative, which is well known for its NMDA receptor antagonism. ${ }^{31}$ The NMDA receptor is found solely on neurons. It is unique in that it requires the binding of two ligands, glutamate and glycine, and membrane depolarization to open its associated ion channel. Ketamine binds to the dizocilpine site on the NMDA receptor, which is usually the site of magnesium ion binding. ${ }^{31}$ Under normal conditions, depolarization causes the removal of the magnesium from the site, which allows for the opening of NMDA ion channels. ${ }^{32,33}$ When these conditions are met, the opening of NMDA receptor ion channels allows for an influx of cations into the neuron, thereby propagating signal transduction cascades. The binding of ketamine thus inhibits the opening of the NMDA channel in a non-competitive manner, preventing signal transduction by the neuron.

The NMDA receptor plays a role in many critical functions of the CNS, most notably excitatory synaptic transmission, plasticity, and neurodegeneration in the central nervous system. ${ }^{32}$ The NMDA receptor is also believed to play an essential role in inducing and maintaining sensitization during pain at each level of the neural axis. ${ }^{20}$ As discussed previously, the number of NMDA receptors 
peripherally and composition of NMDA subunits centrally are believed to be responsive to increased nociceptive signaling, thereby potentiating chronic pain. ${ }^{20}$ In addition, NMDA receptors have also been found to be integral components of visceral pain pathways. ${ }^{20}$ Given the possible role of NMDA receptors in chronic pain networks, and the ability of ketamine to non-competitively inhibit the receptor, there is increased interest in the drug's use to treat chronic pain. ${ }^{27}$ Additionally, it is believed that ketamine may increase central modulation also to inhibit chronic pain pathways. ${ }^{34}$ Nevertheless, given the other important roles NMDA receptors play within the CNS and the negative effects of their inhibition centrally, such as ataxia and memory impairment, ongoing research is attempting to determine more specific ways to target the NMDA receptor. ${ }^{20,32,33,35}$

Ketamine also has clinically significant interactions with other receptors, but these are largely weaker than its NMDA receptor antagonism. ${ }^{31,34}$ Among opioid receptors, ketamine has been found to interact at several receptors - although its exact interaction is still not fully understood. ${ }^{31}$ For example, ketamine is believed to be an antagonist at $\mu$ receptors while an agonist at $\kappa$-opioid receptors. At subanesthetic doses, ketamine has been found to potentiate opioid-analgesia through NMDA antagonism - but at anesthetic concentrations, it has been found to exert antagonistic actions at both $\mu$ and $\kappa$ receptors, possibly. ${ }^{35}$ Other possible effects attributed to ketamine include an inhibitory effect on catecholamine uptake in shock patients, anti-tumor effects through NMDA antagonism, and anti-inflammatory effects by possibly inhibiting transcription factor activator protein-1 (TFAP-1) and nuclear factor- $\kappa \mathrm{B}$ (NFк B). ${ }^{35}$

\section{CLINICAL APPLICABILITY IN CHRONIC PAIN}

The use of ketamine to treat chronic pain has been widely studied among several conditions, including complex regional pain syndrome, fibromyalgia, chronic neuropathic pain, post-thoracotomy pain, cancer pain, and phantom limb pain. The major clinical trials performed to study each of these conditions are discussed below.

\section{COMPLEX REGIONAL PAIN SYNDROME}

Complex regional pain syndrome (CRPS) is a form of chronic limb pain that typically arises following limb trauma. The pain can be categorized as burning, throbbing, or aching and may be combined with swelling, temperature changes, and even structural variations in the skin or bones of the affected limb. Its diagnosis notably excludes cases where a nerve injury is established, even though its clinical presentation may resemble nerve injury. The pathophysiology of CRPS is not well-understood. ${ }^{36}$ Commonly used pain treatments, including NSAIDs, opioids, antidepressants, muscle relaxants, and antiepileptics, have not been proven to be effective in the management of CRPS. ${ }^{37,38}$

Sigtermans et al. studied the use of a 4.2-day continuous infusion of low-dose ketamine in patients with CRPS-1 who responded poorly to standard pain treatment. Low-dose ketamine was able to significantly lower pain scores until week 12 in comparison to placebo. However, there was no difference in functional status between the two groups. ${ }^{38}$ Schwartzman et al. also examined the use of low-dose ketamine infusion in patients with CRPS whose pain was intractable for at least six months. Results showed that a daily 4-hour low-dose ketamine infusion for ten days significantly reduced overall pain, burning pain, and pain to light touch compared to placebo after 12 weeks. Yet, similar to the Sigtermans et al. study, there was no significant difference in activity level, quantitative sensory testing, and quality of life between the ketamine and control groups. 39

Both studies' participants experienced side effects such as headache and nausea, with Sigtermans et al. finding a higher incidence of nausea in the ketamine group. Sigtermans et al. also reported an increased rate of psychomimetic side effects in the ketamine group, with two participants stopping their infusions prematurely to the severity of psychomimetic effects. ${ }^{38}$ Schwartzman et al. did not have any reports of agitation, blurred vision, delusions, hallucinations, or out-of-body experiences in either group. ${ }^{39}$ Despite the reported lack of improvement in quality of life and potential side effects, ketamine's effectiveness in reducing pain for CRPS patients suggests that it should be strongly considered as a treatment option when CRPS pain is refractory to standard treatment.

\section{FIBROMYALGIA}

Fibromyalgia is a generalized pain disorder characterized by widespread pain and tenderness. It is distinguished from CRPS by a lack of association with trauma and a more diffuse area of involvement. Its pathogenesis is not fully understood. ${ }^{40-42}$ Treatment modalities include pharmacologic and non-pharmacologic therapies. Pharmacologic options include tricyclic antidepressants (TCAs), selective serotonin reuptake inhibitors (SSRIs), serotonin-norepinephrine reuptake inhibitors (SNRIs), and antiepileptics. Non-pharmacologic treatments include exercise, massage therapy, and acupuncture. Despite the variety of treatments, their success in reducing fibromyalgia pain is mixed and can vary from patient to patient. ${ }^{41,42}$

Toppers et al. investigated the use of 30-minute relatively high-dose IV ketamine infusions in patients with fibromyalgia, using midazolam infusions for its control group. While ketamine and midazolam both significantly improved pain scores, there was no significant difference in pain relief between the two groups. There was also no significant difference in functional status or quality of life between groups after eight weeks. Toppers et al. also reported a significantly higher incidence of drowsiness and drug high in the ketamine group compared to midazolam. ${ }^{43}$ These results suggest that ketamine is unlikely to be an advisable treatment for fibromyalgia.

\section{CHRONIC NEUROPATHIC PAIN}

Chronic neuropathic pain arises from a lesion or disease affecting the somatosensory system. ${ }^{44}$ It has been described as a tingling, burning, or shooting sensation of pain yet presents with a paradoxical sensory loss in the area of pain. Treatment options include antidepressants (in particular 
TCAs), anticonvulsants, topical anesthetics, and opioids. ${ }^{45,46}$

Vranken et al. studied the use of $50 \mathrm{mg}$ and $75 \mathrm{mg}$ transdermal ketamine patches in patients with central neuropathic pain refractory to standard treatments. Each participant received an iontophoresis patch that continuously delivered medication for 24-hours and was changed daily for five days. There was no significant difference in pain reduction between the placebo and $50 \mathrm{mg}$ and $75 \mathrm{mg}$ ketamine groups one week after treatment introduction. While the quality of life and health status significantly improved in the $75 \mathrm{mg}$ ketamine group compared to placebo, it did not improve in the $50 \mathrm{mg}$ ketamine group 1 week following treatment. The side effects were reportedly mild and transient. The placebo and 50mg ketamine groups had similar cases of sedation, dizziness, vivid dreams, headache, and erythema, while the $75 \mathrm{mg}$ ketamine group reported instances of nausea and vomiting. ${ }^{47}$ Rigo et al. compared the use of oral methadone and oral ketamine in patients with chronic neuropathic pain for at least six months refractory to standard treatment options. Participants received either methadone, ketamine, or methadone plus ketamine for a total of 90 days. All study groups were able to decrease pain scores from baseline by at least $40 \%$. However, the ketamine-only group had a significant reduction in pain scores relative to the other groups. While there was no significant reduction in burning pain with any groups, there was a significant reduction in allodynia in the ketamine-only group. The incidence of side effects was similar between all groups, but there was a significantly higher incidence of somnolence in the methadone-only group compared to the other groups. ${ }^{48}$

An alternative approach to treating pain is the employment of conditioned pain modulation (CPM), the inhibition of neuropathic pain using a noxious stimulus in a remote area. Niesters et al. studied the use of IV ketamine and morphine in conjunction with CPM in patients with neuropathic pain caused by peripheral neuropathy. Participants received infusions of ketamine, morphine, or normal saline. All participants received CPM before treatment and 20 minutes after. CPM was performed by immersing an unaffected leg in cold water. The ketamine and morphine groups had significant reductions in pain in conjunction with CPM, while the control group did not. The magnitude of pain reduction did not differ between the ketamine and morphine groups, although the ketamine group experienced a longer pain relief than the morphine group. The only reported side effect was nausea, which was more common in the morphine group than the ketamine group. ${ }^{34}$

Magnesium sulfate is a physiological blocker of NMDA receptors used in combination with ketamine to treat pain following scoliosis surgery and bariatric surgery. ${ }^{49,50}$ Pickering et al. studied the use of ketamine and magnesium for patients with chronic neuropathic pain refractory to standard treatment. A within-subjects design was used so that each participant received IV ketamine, co-administration of IV ketamine and magnesium, and IV normal saline in a random order every 35 days. They found no significant difference in pain intensity, emotional status, sleep, quality of life, or patient satisfaction between the three treatments. Nausea and fatigue were reported in all three treatment op- tions, and ketamine-only and ketamine-magnesium groups also reported feelings of drunkenness, headache, high blood pressure, dizziness, and hot flashes. ${ }^{51}$

Amr examined chronic neuropathic pain in patients with spinal cord injuries. Participants received oral gabapentin and either a daily 5-hour infusion of ketamine or placebo for seven days. There was a significant reduction in pain scores in the ketamine-gabapentin group until week 3 of the 1-month study, at which point there was no significant difference in pain. Patients from both gabapentin-only and ketamine-gabapentin groups endorsed dizziness, tiredness, and lack of coordination, and few patients receiving ketamine endorsed short-lasting delusions after infusions. ${ }^{52}$

Looking into a different subset of patients, Mitchell \& Fallon studied IV ketamine for the treatment of neuropathic pain caused by critical limb ischemia secondary to peripheral vascular disease. Participants were continued on the treatment regimen they followed before enrollment and then received either a 4-hour infusion of ketamine or placebo for one day. The ketamine group reported a significant improvement in pain relief across the duration of the 5-day study. The ketamine group also significantly improved activity levels and enjoyment of life compared to the control group. However, there was no difference in total opioid requirements within 24-hours between the two groups. The ketamine group experienced a higher incidence of heightened emotions, although there was no difference in instances of nausea between both groups. ${ }^{53}$

Overall, these results indicate that ketamine should be considered a pharmacologic option for chronic neuropathic pain refractory to standard treatment. The positive effect of its potential pain reduction should be weighed in conjunction with the variety of side effects it may cause and the etiology of the patient's neuropathic pain.

\section{POST-THORACOTOMY PAIN SYNDROME}

Post-thoracotomy pain syndrome (PTPS) is pain that persists or recurs along the thoracostomy incision for at least two months following surgery. ${ }^{54}$ It resembles neuropathic pain and is characterized by burning pain with localized tenderness around the incision site. PTPS can also affect respiratory activity by restricting sputum clearance and ventilatory capacity, affecting long-term patient outcomes. Current management involves intra-operative thoracic epidural analgesia with a local anesthetic or potent opioid. The aim is to prevent the processing of pain before the surgical insult. 55

Mendola et al. studied the use of a 60-hour ketamine infusion as an adjuvant to thoracic epidural analgesia in patients undergoing thoracostomy. The ketamine infusion resulted in a significant reduction in pain compared to placebo during the first month following the procedure. However, there was no significant difference in pain control between ketamine and control groups during months 3 and 6. ${ }^{56}$ Joseph et al. also investigated ketamine as an adjuvant to thoracic epidural analgesia in patients undergoing thoracotomy, but with a 48-hour infusion. There was no significant difference between the two groups in consumption of analgesics, early postoperative pain, pulmonary function, or residual pain. ${ }^{25}$ Dualé et al. investigated the use of ke- 
tamine as an adjuvant to intrapleural ropivacaine in patients undergoing thoracostomy. Ketamine provided significant immediate postoperative pain control, but there was no difference in neuropathic pain or intake of analgesics at six weeks and four months after surgery. ${ }^{57}$ Side effects were wide-ranging in all three studies, but there was no significant difference in incidence. These studies suggest that ketamine is not a particularly effective adjuvant treatment to prevent PTPS.

\section{CANCER PAIN}

Cancer pain can derive from cancer itself, such as bone metastasis or tumor mass effect, or treatment-related adverse effects, such as chemotherapy-induced peripheral neuropathy, chemotherapy- or radiation-induced mucositis, and postoperative wound pain. The mainstay of pharmacologic treatment options includes NSAIDs for mild to moderate pain, opioids for more severe somatic pain, and antiepileptics for neuropathic pain, with antidepressants, anxiolytics, and steroids sometimes used supplementarily. Procedural interventions like radiation for bone metastasis and nerve blocks are typically performed when a patient's pain is proven refractory to pharmacologic therapy. ${ }^{58}$

Unfortunately, there is a limited significant clinical application for ketamine in relieving cancer pain. Hardy et al. found no clinical advantage in the administration of subcutaneous ketamine compared to placebo. Pain scores in both treatment groups improved over time but were not significantly different. Furthermore, the ketamine group experienced a more significant increase in toxicity. More patients withdrew from the treatment group due to this toxicity and the greater occurrence of adverse events such as psychotoxicity and injection site reactions. ${ }^{59}$ The lack of significant pain reduction compared to placebo combined with the risk of side effects and toxicity suggest that ketamine should not be administered to treat chronic cancer pain. These results carry over into the category of cancer pain in the palliative care population. Salas et al. observed that palliative care patients had no significant improvement in pain with the use of IV ketamine in addition to morphine in comparison to a control group of morphine alone. They hypothesized that this was most likely due to patients' long histories with pain-relieving treatment that may have caused altered responsiveness and metabolism of ketamine. In contrast to the non-palliative cancer patient group in Hardy et al., the co-administration of ketamine in palliative care cancer patients was not associated with an increase in adverse events. ${ }^{60}$

\section{PHANTOM LIMB PAIN}

Phantom limb pain (PLP) is a form of chronic pain occurring in a body part no longer present. It appears in over half of all amputees and is usually characterized as neuropathic pain. ${ }^{61}$ There is no established prevention modality, as perioperative epidural analgesia and ketamine failed to prevent its development. ${ }^{62,63}$ For treatment, RCTs have shown mixed results with gabapentin and positive results with oral and IV morphine. ${ }^{64-67}$

Nikolajsen et al. found a decrease in PLP with IV keta- mine when compared to placebo. Ketamine reduced windup-like pain (pain from repeatedly tapping the skin area with abnormal sensation) and increased the threshold for pressure pain. ${ }^{68}$ Following this study, other drugs acting as NMDA antagonists were investigated for the treatment of PLP. Dextromethorphan was found to be effective, but memantine was not. ${ }^{69-72}$ A crossover study also demonstrated PLP reduction with calcitonin. ${ }^{73}$ Eichenberger et al. studied ketamine and calcitonin as treatment modalities. They found that ketamine significantly reduced PLP, calcitonin, and placebo did not, and co-administration of ketamine and calcitonin was not significantly different from ketamine alone in reducing pain intensity. However, only the combination of ketamine and calcitonin improved average and peak pain intensity 48 -hours after treatment. ${ }^{74}$ These results support the administration of ketamine infusions for the reduction of phantom limb pain.

\section{DEPRESSION, A COMPONENT OF CHRONIC PAIN}

Ketamine has been found to play a role in relieving depressive symptoms. Unlike other antidepressants that require weeks to months of continuous therapy, the effects of IV ketamine can be observed hours to days after treatment. ${ }^{75,76}$ In fact, Zarate et al. found that ketamine can improve depressive symptoms in as little as 110 minutes and can last up to one week after a single infusion. ${ }^{76}$ It should be noted that despite ketamine's week-long effects, its antidepressive action was not significantly different from midazolam. ${ }^{77}$ The mechanism by which ketamine alleviates depressive symptoms was unknown until Li et al. determined a connection to the mammalian target of rapamycin (mTOR). More specifically, ketamine was found to activate mTOR in the prefrontal cortex of mouse models quickly. Other antidepressants in this study, such as imipramine and fluoxetine, did not impact mTOR signaling, highlighting ketamine's novel mechanism of action in modulating depression. This increase in mTOR signaling allowed for increased synthesis in pre-and post-synaptic proteins that allow for new synapse and spine formation within 24-hours in the prefrontal cortex of mice. Since depression causes atrophy and degeneration of pyramidal neurons in the prefrontal cortices, this may be the main mechanism by which ketamine treats depression. ${ }^{78}$

In addition to the chronic treatment of depression, ketamine can also be used in the acute setting of suicidal ideations and attempts. Ligeti et al. found that an IV ketamine push of $0.5 \mathrm{mg} / \mathrm{kg}$ effectively reverses depressive and suicidal symptoms post-suicide attempt within minutes. The effects of pushed ketamine lasted up to 120 hours and continued to have anti-depressive/anti-suicidal results until patient follow-up six months later. Therefore, ketamine pushes could play a significant role in acute remission of the most severe forms of depression. ${ }^{79}$

In addition to ketamine's efficacy in treating chronic depression, its safety must also be considered. The two most common side effects of ketamine are psychomimetic effects and dissociation. Patients have also reported a feeling of "being high" after an initial dose of ketamine. As mentioned earlier, other side effects of clinical doses include nausea, vomiting, drowsiness, confusion, somnolence, and 
headache. More severe side effects can occur if ketamine is misused, which has been a common occurrence since the emergence of ketamine as a street drug in the 1960s. Ketamine's addictive properties can lead to its use in large doses, yielding severe side effects such as white matter changes, memory changes, neurocognitive impairment, and reduced well-being. Damage to the urinary system has also been noted when ketamine is used in large doses for prolonged periods of time. Ketamine's clinical and abusive dosing side effects along with its misuse potential should be considered before starting patients on this experimental medication, particularly patients clinically diagnosed with depression. ${ }^{77}$

\section{NMDA ANTAGONISTS AND MORPHINE HYPERALGESIA}

Morphine hyperalgesia is a paradoxical side effect of opioids. It is characterized by the increased sensitization to painful stimuli while receiving opioid therapy. The mechanism by which opioids produce hyperalgesia is paradoxical as well, in that hyperalgesia is not mediated by the same mu receptors that trigger analgesia. Juni et al. and van Dorp et al. found that hyperalgesia persisted in mice treated with continuous intravenous morphine infusions despite abolishing morphine's analgesic effects via the addition of the opioid mu receptor antagonist, naltrexone. Hyperalgesia increased in mice receiving naltrexone, indicating that only analgesia, not hyperalgesia, is generated by the mu receptor. In these studies, the NMDA receptor antagonist MK-801 effectively abolished hyperalgesia in morphine-treated mice, indicating that this receptor plays a key role. Research speculates that NMDA antagonism diminishes hyperalgesia via potentiating morphine analgesia and has been supported by these experiments displaying that co-administration of naltrexone with MK-801 reduces hyperalgesia. ${ }^{80,81}$

\section{KETAMINE AND OPIOID USE}

Ketamine has been studied in conjunction with opioid use due to its antagonism of the NMDA receptor, responsible for tolerance and hyperalgesia. Researchers are hopeful that in reducing pain and potentiating morphine analgesia, ketamine usage will reduce opioid requirements in chronic pain patients. Barreveld et al. observed that continuous IV ketamine infusion in postoperative patients until hospital discharge led to decreased average pain in chronic opioid users. However, administering ketamine until discharge did not reduce the amount of opioid consumption postoperatively in this population. ${ }^{82}$ Other researchers have studied ketamine's benefit in chronic opioid users beyond the immediate postoperative period. Nielsen et al. showed that a perioperative IV ketamine bolus had long-term advantages for chronic opioid users undergoing surgery. The ketamine group reported less daily use of opioids or any other anal- gesics and better mobilization pain, pain at rest, and labor market attachment. ${ }^{83}$ Beyond the surgical setting, ketamine has also been used for pain control in critically ill trauma patients. Prusowski et al. showed that continuous ketamine infusions for sedation and agitation were associated with less opioids and propofol required to manage these patients.

In contrast, this same study found that ketamine infusion was shown to increase the use of ziprasidone and dexmedetomidine. ${ }^{84}$ This data indicates that ketamine displays the potential to alleviate pain in chronic opioid users further and improve their quality of life. However, it is still not definitively proven to reduce opioid requirements and therefore may leave patients vulnerable to the side effects of other pain medications they may be taking.

\section{CONCLUSION}

Chronic pain affects roughly $20 \%$ of the population worldwide, and many patients experience its detrimental effects on their quality of life and mental health. Ketamine, an NMDA antagonist that also acts on opioid receptors, has been studied as a pharmacologic option for chronic pain conditions. RCTs have shown that ketamine is an effective treatment modality for CRPS and PLP refractory to standard treatment options and should be considered for refractory mixed neuropathic pain. It has also been found to be an effective long-term pharmacologic therapy for depression, a common comorbidity of chronic pain that may affect its disease course. Nonetheless, it is important to weigh its pain-reducing properties with its potential adverse effects, notably psychomimetic and dissociative effects. While it has yet to be successfully demonstrated clinically, ketamine also has the potential to reduce opioid requirements, both from its pain-reducing properties as well as its ability to diminish morphine hyperalgesia. However, it should be noted that the research performed on ketamine to treat chronic pain has its limitations. Most significantly, there is a relative shortage of RCTs studying its effectiveness for treating chronic pain conditions.

Moreover, some of these studies had inadequate blinding, a lack of large participant populations and statistical power, and short follow-up periods. Yet, because ketamine is widely available and inexpensive relative to other pharmacologic therapies for chronic refractory pain, it is likely that researchers will continue to investigate its efficacy and optimal dosage and administration in the future. As the opioid crisis in the United States continues to persist, a better understanding of alternative drug therapies such as ketamine can improve how chronic pain is managed and offer additional treatment options for patients.

Submitted: June 30, 2021 EST, Accepted: July 05, 2021 EST 


\section{REFERENCES}

1. Treede RD, Rief W, Barke A, et al. A classification of chronic pain for ICD-11. Pain. 2015;156(6):1003-1007. doi:10.1097/i.pain.0000000000000160

2. Johannes CB, Le TK, Zhou X, Johnston JA, Dworkin $\mathrm{RH}$. The Prevalence of Chronic Pain in United States Adults: Results of an Internet-Based Survey. J Pain. 2010;11(11):1230-1239. doi:10.1016/j.jpain.2010.07.0 02

3. Treede RD, Rief W, Barke A, et al. Chronic pain as a symptom or a disease: The IASP Classification of Chronic Pain for the International Classification of Diseases (ICD-11). Pain. 2019;160(1):19-27. doi:10.10 97/i.pain.0000000000001384

4. Dworkin RH, O'Connor AB, Audette J, et al. Recommendations for the pharmacological management of neuropathic pain: an overview and literature update. Mayo Clin Proc. 2010;85(3 Suppl):S3-S14. doi:10.4065/mcp.2009.0649

5. Nicholas M, Vlaeyen JWS, Rief W, et al. The IASP classification of chronic pain for ICD-11: Chronic primary pain. Pain. 2019;160(1):28-37. doi:10.1097/j.p ain.0000000000001390

6. Dureja GP, Jain PN, Shetty N, et al. prevalence of chronic pain, impact on daily life, and treatment practices in India. Pain Pract. 2014;14(2):E51-E62. do i:10.1111/papr.12132

7. Van Hecke O, Torrance N, Smith BH. Chronic pain epidemiology and its clinical relevance. Br J Anaesth. 2013;111(1):13-18. doi:10.1093/bja/aet123

8. Dahlhamer J, Lucas J, Zelaya C, et al. Prevalence of Chronic Pain and High-Impact Chronic Pain Among Adults - United States, 2016. MMWR Morb Mortal Wkly Rep. 2018;67(36):1001-1006. doi:10.15585/mmw r.mm6736a2

9. Zorina-Lichtenwalter K, Meloto CB, Khoury S, Diatchenko L. Genetic predictors of human chronic pain conditions. Neuroscience. 2016;338:36-62. doi:1 $\underline{0.1016 / \text { i.neuroscience.2016.04.041 }}$

10. Mills SEE, Nicolson KP, Smith BH. Chronic pain: a review of its epidemiology and associated factors in population-based studies. Br J Anaesth. 2019;123(2):e273-e283. doi:10.1016/i.bja.2019.03.023

11. Wiesenfeld-Hallin Z. Sex differences in pain perception. Gend Med. 2005;2(3):137-145. doi:10.101 6/s1550-8579(05)80042-7
12. Sachs-Ericsson N, Kendall-Tackett K, Hernandez A. Childhood abuse, chronic pain, and depression in the National Comorbidity Survey. Child Abuse Negl. 2007;31(5):531-547. doi:10.1016/j.chiabu.2006.12.007

13. Jank R, Gallee A, Boeckle M, Fiegl S, Pieh C. Chronic Pain and Sleep Disorders in Primary Care. Pain Res Treat. 2017;2017:9081802. doi:10.1155/2017/ $\underline{9081802}$

14. Costigan M, Scholz J, Woolf CJ. Neuropathic pain: a maladaptive response of the nervous system to damage. Annu Rev Neurosci. 2009;32(1):1-32. doi:10.1 146/annurev.neuro.051508.135531

15. Woolf CJ. Central sensitization: implications for the diagnosis and treatment of pain. Pain. 2011;152(3 Suppl):S2-S15. doi:10.1016/i.pain.2010.09.030

16. Johnson MI. The physiology of the sensory dimensions of clinical pain. Physiotherapy. 1997;83(10):526-536. doi:10.1016/s0031-9406(05)656 10-6

17. Fields HL, Basbaum AI, Heinricher MM. Central nervous system mechanisms of pain modulation. In: Wall and Melzack's Textbook of Pain. Elsevier; 2006:125-142. https://doi.org/10.1016/b0-443-0728 7-6/50012-6

18. Ossipov MH, Dussor GO, Porreca F. Central modulation of pain. J Clin Invest. 2010;120(11):3779-3787. doi:10.1172/jci43766

19. Petrenko AB, Yamakura T, Baba H, Shimoji K. The role of N-methyl-D-aspartate (NMDA) receptors in pain: A review. Anesth Analg. 2003;97(4):1108-1116. d oi:10.1213/01.ane.0000081061.12235.55

20. Carlton SM, Coggeshall RE. Inflammationinduced changes in peripheral glutamate receptor populations. Brain Res. 1999;820(1-2):63-70. doi:10.1 016/s0006-8993(98)01328-6

21. Gaunitz C, Schüttler A, Gillen C, Allgaier C. Formalin-induced changes of NMDA receptor subunit expression in the spinal cord of the rat. Amino Acids. 2002;23(1-3):177-182. doi:10.1007/s00726-001-012 $\underline{5-3}$

22. Jansen KLR. A review of the nonmedical use of ketamine: Use, users and consequences. J Psychoactive Drugs. 2000;32(4):419-433. doi:10.1080/02791072.200 0.10400244 
23. Morris H, Wallach J. From PCP to MXE: a comprehensive review of the non-medical use of dissociative drugs. Drug Test Anal. 2014;6(7-8):614-632. doi:10.1002/dta.1620

24. Kronenberg RH. Ketamine as an analgesic: parenteral, oral, rectal, subcutaneous, transdermal and intranasal administration. J Pain Palliat Care Pharmacother. 2002;16(3):27-35. doi:10.1080/j354v16 $\underline{\mathrm{n} 03 \_3}$

25. Domino EF. Taming the ketamine tiger. Anesthesiology. 2010;113(3):678-684. doi:10.1097/al n.0b013e3181ed09a2

26. Schwenk ES, Viscusi ER, Buvanendran A, et al. Consensus Guidelines on the Use of Intravenous Ketamine Infusions for Acute Pain Management From the American Society of Regional Anesthesia and Pain Medicine, the American Academy of Pain Medicine, and the American Society of Anesthesiologists. Reg Anesth Pain Med. 2018;43(5):456-466. doi:10.1097/aap.0000000000000 $\underline{806}$

27. Cohen SP, Bhatia A, Buvanendran A, et al. Consensus Guidelines on the Use of Intravenous Ketamine Infusions for Chronic Pain From the American Society of Regional Anesthesia and Pain Medicine, the American Academy of Pain Medicine, and the American Society of Anesthesiologists. Reg Anesth Pain Med. 2018;43(5):521-546. doi:10.1097/aa p.0000000000000808

28. Sanacora G, Frye MA, McDonald W, et al. A Consensus Statement on the Use of Ketamine in the Treatment of Mood Disorders. JAMA Psychiatry. 2017;74(4):399-405. doi:10.1001/jamapsychiatry.201 $\underline{7.0080}$

29. Caddy C, Amit BH, McCloud TL, et al. Ketamine and other glutamate receptor modulators for depression in adults. Cochrane Database Syst Rev. 2015;(9):CD011612. doi:10.1002/14651858.cd01161 2.pub2

30. Kim J, Farchione T, Potter A, Chen Q, Temple R. Esketamine for Treatment-Resistant Depression First FDA-Approved Antidepressant in a New Class. $N$ Engl J Med. 2019;381(1):1-4. doi:10.1056/nejmp19033 $\underline{05}$

31. Hirota K, Lambert DG. Ketamine: its mechanism(s) of action and unusual clinical uses. $\mathrm{Br} \mathrm{J}$ Anaesth. 1996;77(4):441-444. doi:10.1093/bja/77.4.44 $\underline{1}$

32. Furukawa H, Singh SK, Mancusso R, Gouaux E. Subunit arrangement and function in NMDA receptors. Nature. 2005;438(7065):185-192. doi:10.10 38/nature04089
33. Monyer H, Sprengel R, Schoepfer R, et al. Heteromeric NMDA receptors: molecular and functional distinction of subtypes. Science. 1992;256(5060):1217-1221. doi:10.1126/science.256.5 060.1217

34. Niesters M, Aarts L, Sarton E, Dahan A. Influence of ketamine and morphine on descending pain modulation in chronic pain patients: A randomized placebo-controlled cross-over proof-of-concept study. Br J Anaesth. 2013;110(6):1010-1016. doi:10.1093/bja/ $\underline{\text { aes578 }}$

35. Hirota K, Lambert DG. Ketamine: new uses for an old drug? Br J Anaesth. 2011;107(2):123-126. doi:10.1 093/bja/aer221

36. Bruehl S. Complex regional pain syndrome. BMJ. 2015;351:h2730. doi:10.1136/bmj.h2730

37. Schwartzman RJ. New treatments for reflex sympathetic dystrophy. $N$ Engl J Med. 2000;343(9):654-656. doi:10.1056/nejm200008313430 911

38. Sigtermans MJ, van Hilten JJ, Bauer MCR, et al. ketamine produces effective and long-term pain relief in patients with Complex Regional Pain Syndrome Type 1. Pain. 2009;145(3):304-311. doi:10.1016/j.pai $\underline{\text { n.2009.06.023 }}$

39. Schwartzman RJ, Alexander GM, Grothusen JR, Paylor T, Reichenberger E, Perreault M. Outpatient intravenous ketamine for the treatment of complex regional pain syndrome: A double-blind placebocontrolled study. Pain. 2009;147(1-3):107-115. doi:1 0.1016/j.pain.2009.08.015

40. Lee JY, Choi SH, Park KS, et al. comparison of complex regional pain syndrome and fibromyalgia. Medicine. 2019;98(7):e14452. doi:10.1097/md.000000 0000014452

41. Chinn S, Caldwell W, Gritsenko K. Fibromyalgia Pathogenesis and Treatment Options Update. Curr Pain Headache Rep. 2016;20(4):25. doi:10.1007/s1191 6-016-0556-x

42. Clauw DJ. Fibromyalgia. JAMA.

2014;311(15):1547. doi:10.1001/jama.2014.3266

43. Noppers I, Niesters M, Swartjes M, et al. Absence of long-term analgesic effect from a short-term Sketamine infusion on fibromyalgia pain: a randomized, prospective, double-blind, active placebo-controlled trial. Eur J Pain. 2011;15(9):942-949. doi:10.1016/j.ejpain.2011.03.008 
44. Scholz J, Finnerup NB, Attal N, et al. The IASP classification of chronic pain for ICD-11: chronic neuropathic pain. Pain. 2019;160(1):53-59. doi:10.109 7/j.pain.0000000000001365

45. Jensen TS, Madsen CS, Finnerup NB.

Pharmacology and treatment of neuropathic pains. Curr Opin Neurol. 2009;22(5):467-474. doi:10.1097/wc o.0b013e3283311e13

46. Park HJ, Moon DE. Pharmacologic Management of Chronic Pain. Korean J Pain. 2010;23(2):99-108. doi:1 $\underline{0.3344 / \mathrm{kjp} .2010 .23 .2 .99}$

47. Vranken JH, Dijkgraaf MGW, Kruis MR, Van Dasselaar NT, Van Der Vegt MH. Iontophoretic administration of $\mathrm{S}(+)$-ketamine in patients with intractable central pain: A placebo-controlled trial. Pain. 2005;118(1-2):224-231. doi:10.1016/j.pain.200 5.08.020

48. Rigo FK, Trevisan G, Godoy MC, et al. Management of neuropathic chronic pain with methadone combined with ketamine: a randomized, double-blind, active-controlled clinical trial. Pain Phys. 2017;20(3):207-215. doi:10.36076/ppj.2017.215

49. Jabbour HJ, Naccache NM, Jawish RJ, et al. Ketamine and magnesium association reduces morphine consumption after scoliosis surgery: Prospective randomised double-blind study. Acta Anaesthesiol Scand. 2014;58(5):572-579. doi:10.1111/ aas. 12304

50. Jabbour H, Jabbour K, Abi Lutfallah A, et al. Magnesium and Ketamine Reduce Early Morphine Consumption After Open Bariatric Surgery: a Prospective Randomized Double-Blind Study. Obes Surg. 2020;30(4):11695-11019. doi:10.1007/s11695-01 9-04317-1

51. Pickering G, Pereira B, Morel V, et al. Ketamine and Magnesium for Refractory Neuropathic Pain: A Randomized, Double-blind, Crossover Trial. Anesthesiol. 2020;133(1):154-164. doi:10.1097/aln.00 $\underline{00000000003345}$

52. Amr YM. Multi-day low dose ketamine infusion as adjuvant to oral gabapentin in spinal cord injuryrelated chronic pain: A prospective, randomized, double-blind trial. Pain Phys. 2010;13(3):245-249. do i:10.36076/ppj.2010/13/245

53. Mitchell AC, Fallon MT. A single infusion of intravenous ketamine improves pain relief in patients with critical limb ischaemia: Results of a double-blind randomised controlled trial. Pain. 2002;97(3):275-281. doi:10.1016/s0304-3959(02)0003 $\underline{3-7}$
54. International Association for the Study of Pain, Subcommittee on Taxonomy. Classification of chronic pain. Descriptions of chronic pain syndromes and definitions of pain terms. Pain Suppl. 1986;3:S1-S226.

55. Moyse DW, Kaye AD, Diaz JH, Qadri MY, Lindsay D, Pyati S. Perioperative ketamine administration for thoracotomy pain. Pain Phys. 2017;20(3):173-184. do $\mathrm{i}: 10.36076 /$ ppj.2017.184

56. Mendola C, Cammarota G, Netto R, et al. S(+)ketamine for control of perioperative pain and prevention of post-thoracotomy pain syndrome: A randomized, double-blind study. Minerva Anestesiol. 2012;78(7).

57. Dualé C, Sibaud F, Guastella V, et al. Perioperative ketamine does not prevent chronic pain after thoracotomy. Eur J Pain. 2009;13(5):497-505. doi:10.1 016/j.ejpain.2008.06.013

58. Deng G. Integrative Medicine Therapies for Pain Management in Cancer Patients. Cancer J. 2019;25(5):343-348. doi:10.1097/ppo.0000000000000 $\underline{399}$

59. Hardy J, Quinn S, Fazekas B, et al. Randomized, double-blind, placebo-controlled study to assess the efficacy and toxicity of subcutaneous ketamine in the management of cancer pain. J Clin Oncol. 2012;30(29):3611-3617. doi:10.1200/ico.2012.42.1081

60. Salas S, Frasca M, Planchet-Barraud B, et al. Ketamine analgesic effect by continuous intravenous infusion in refractory cancer pain: Considerations about the clinical research in palliative care. J Palliat Med. 2012;15(3):287-293. doi:10.1089/jpm.2011.0353

61. Flor H. Phantom-limb pain: characteristics, causes, and treatment. Lancet Neurol. 2002;1(3):182-189. doi:10.1016/s1474-4422(02)0007 4-1

62. Nikolajsen L, Ilkjaer S, Christensen JH, Krøner K, Jensen TS. Randomised trial of epidural bupivacaine and morphine in prevention of stump and phantom pain in lower-limb amputation. Lancet. 1997;350(9088):1353-1357. doi:10.1016/s0140-6736(9 7)06315-0

63. Hayes C, Armstrong-Brown A, Burstal R. Perioperative intravenous ketamine infusion for the prevention of persistent post-amputation pain: a randomized, controlled trial. Anaesth Intensive Care. 2004;32(3):330-338. doi:10.1177/0310057x040320030 $\underline{5}$ 
64. Bone M, Critchley P, Buggy DJ. Gabapentin in postamputation phantom limb pain: A randomized, double-blind, placebo-controlled, cross-over study. Reg Anesth Pain Med. 2002;27(5):481-486. doi:10.105 3/rapm.2002.35169

65. Nikolajsen L, Finnerup NB, Kramp S, Vimtrup AS, Keller J, Jensen TS. A randomized study of the effects of gabapentin on postamputation pain.

Anesthesiology. 2006;105(5):1008-1015. doi:10.1097/0 $\underline{0000542-200611000-00023}$

66. Wu CL, Tella P, Staats PS, et al. Analgesic effects of intravenous lidocaine and morphine on postamputation pain: a randomized double-blind, active placebo-controlled, crossovercrossover trial. Anesthesiology. 2002;96(4):841-848. doi:10.1097/0000 0542-200204000-00010

67. Huse E, Larbig W, Flor H, Birbaumer N. The effect of opioids on phantom limb pain and cortical reorganization. Pain. 2001;90(1-2):47-55. doi:10.101 6/s0304-3959(00)00385-7

68. Nikolajsen L, Hansen CL, Nielsen J, Keller J, Arendt-Nielsen L, Jensen TS. The effect of ketamine on phantom pain: a central neuropathic disorder maintained by peripheral input. Pain. 1996;67(1):69-77. doi:10.1016/0304-3959(96)03080-1

69. Ben Abraham R, Marouani N, Weinbroum AA. Dextromethorphan mitigates phantom pain in cancer amputees. Ann Surg Oncol. 2003;10(3):268-274. doi:1 $\underline{0.1245 / \text { aso.2003.08.007 }}$

70. Nikolajsen L, Gottrup H, Kristensen AGD, Jensen TS. Memantine (a N-methyl-D-aspartate receptor antagonist) in the treatment of neuropathic pain after amputation or surgery: a randomized, doubleblinded, crossover study. Anesth Analg. 2000;91(4):960-966. doi:10.1097/00000539-20001000 $\underline{0-00036}$

71. Maier C, Dertwinkel R, Mansourian N, et al. Efficacy of the NMDA-receptor antagonist memantine in patients with chronic phantom limb pain - results of a randomized double-blinded, placebo-controlled trial. Pain. 2003;103(3):277-283. doi:10.1016/s0304-3 959(02)00456-6

72. Wiech K, Kiefer RT, T??pfner S, et al. A PlaceboControlled Randomized Crossover Trial of the NMethyl-d-Aspartic Acid Receptor Antagonist, Memantine, in Patients with Chronic Phantom Limb Pain. Anesth Analg. Published online February 2004:408-413. doi:10.1213/01.ane.0000096002.5381 8.bd

73. Jaeger H, Maier C. Calcitonin in phantom limb pain: a double-blind study. Pain. 1992;48(1):21-27. do i:10.1016/0304-3959(92)90127-w
74. Eichenberger U, Neff F, Sveticic G, et al. Chronic phantom limb pain: The effects of calcitonin, ketamine, and their combination on pain and sensory thresholds. Anesth Analg. 2008;106(4):1265-1273. do $\mathrm{i}: 10.1213 /$ ane.0b013e3181685014

75. Berman RM, Cappiello A, Anand A, et al. Antidepressant Effects of Ketamine in Depressed Patients. Biol Psychiatry. 2000;47(4):351-354. doi:10.1 016/s0006-3223(99)00230-9

76. Zarate CA, Singh JB, Carlson PJ, et al. A randomized trial of an N-methyl-D-aspartate antagonist in treatment-resistant major depression. Arch Gen Psychiatry. 2006;63(8):856-864. doi:10.1001/ archpsyc.63.8.856

77. Schoevers RA, Chaves TV, Balukova SM, aan Het Rot M, Kortekaas R. Oral ketamine for the treatment of pain and treatment-resistant depression. $\mathrm{Br} \mathrm{J}$ Psychiatry. 2016;208(2):108-113. doi:10.1192/bjp.bp.1 15.165498

78. Li N, Lee B, Liu RJ, et al. mTOR-dependent synapse formation underlies the rapid antidepressant effects of NMDA antagonists. Science.

2010;329(5994):959-964. doi:10.1126/science.119028 $\underline{7}$

79. Aligeti S, Quinones M, Salazar R. Rapid Resolution of Suicidal Behavior and Depression With Single Low-Dose Ketamine Intravenous Push Even After 6 Months of Follow-Up. J Clin Psychopharmacol. 2014;34(4):533-535. doi:10.1097/jcp.00000000000001 $\underline{46}$

80. Juni A, Klein G, Kest B. Morphine hyperalgesia in mice is unrelated to opioid activity, analgesia, or tolerance: Evidence for multiple diverse hyperalgesic systems. Brain Res. 2006;1070(1):35-44. doi:10.1016/ j.brainres.2005.11.054

81. Van Dorp ELA, Kest B, Kowalczyk WJ, et al. Morphine- $6 \beta$-glucuronide rapidly increases pain sensitivity independently of opioid receptor activity in mice and humans. Anesthesiology. 2009;110(6):1356-1363. doi:10.1097/aln.0b013e3181a 105de

82. Barreveld AM, Correll DJ, Liu X, et al. ketamine decreases postoperative pain scores in patients taking opioids for chronic pain: Results of a prospective, randomized, double-blind study. Pain Med. 2013;14(6):925-934. doi:10.1111/pme.12086

83. Nielsen RV, Fomsgaard JS, Nikolajsen L, Dahl JB, Mathiesen O. Intraoperative S-ketamine for the reduction of opioid consumption and pain one year after spine surgery: A randomized clinical trial of opioid-dependent patients. Eur J Pain. 2019;23(3):455-460. doi:10.1002/ejp.1317 
84. Pruskowski KA, Harbourt K, Pajoumand M, Chui $\mathrm{SHJ}$, Reynolds HN. Impact of Ketamine Use on Adjunctive Analgesic and Sedative Medications in Critically Ill Trauma Patients. Pharmacother. 2017;37(12):1537-1544. doi:10.1002/phar.2042 